\title{
O IMPACTO DO TRANSPLANTE PEDIÁTRICO NA FAMÍLIA: REVISÃO DA LITERATURA
}

\author{
The impact of the pediatric transplant on the family: a review of the literature
}

Ana Márcia Chiaradia Mendes, Maria Clara Cassuli Matheus, Regina Szylit Bousso

\section{RESUMO}

Objetivo: explorar o conhecimento disponível na literatura de ciências da saúde sobre a experiência das famílias de crianças submetidas a transplante de órgãos, bem como identificar lacunas nessa área. Trata-se de uma revisão sistemática da literatura, em que os textos foram selecionados nas bases de dados Medline, Pubmed e Lilacs, utilizando as palavras-chave child, transplantation e family. Os nove textos selecionados para análise foram organizados quanto ao tipo de estudo, ano e local de publicação, objetivos, método e principais resultados. A análise dos trabalhos permitiu identificar quatro categorias principais abordadas na literatura: Funcionamento e Adaptação Psicossocial, Experiência familiar, Qualidade de vida e Indicadores de prognóstico, sendo a totalidade das publicações de origem internacional. Concluise que a literatura já traz dados que comprovam o grande impacto que a experiência do transplante exerce sobre a criança e sua família, porém, pesquisas futuras que enfoquem a experiência da família em termos de sua dinâmica em todo o processo do transplante e abordando intervenções de Enfermagem centradas no cuidado a essas famílias fazem-se necessárias.

Descritores: Literatura de Revisão como Assunto; Transplantes; Família; Relações Familiares.

Instituição:

Escola de Enfermagem da Universidade de São Paulo - EEUSP; Departamento de Enfermagem da Universidade Federal de São Paulo - UNIFESP.

\section{Correspondência:}

Ana Márcia Chiaradia Mendes

Rua Zacarias de Góis, 1326 apto 41- Campo Belo

CEP 04610-003 - São Paulo - SP

Tels.: 11 5535-0547/8644-6164

E-mail:mendes_amc@yahoo.com.br

Recebido em: 25.05.2007

Aceito em: 25.06.2007

\section{INTRODUÇÃO}

O transplante de órgãos não é mais considerado um procedimento experimental, mas uma intervenção cirúrgica amplamente aceita e recomendada para o tratamento de disfunções degenerativas e progressivas de órgãos de crianças e adultos, principalmente com doenças renais, hepáticas, pulmonares e cardíacas, por proporcionar aumento na sobrevida desses pacientes e melhor qualidade de vida. ${ }^{1-5}$

Avanços contínuos têm sido feitos, principalmente no que diz respeito ao aprimoramento da técnica cirúrgica e aos mecanismos mais eficientes de imunossupressão, de forma a aumentar cada vez mais a sobrevida e a qualidade de vida dos pacientes transplantados. A literatura traz extensos exemplos sobre os principais avanços, dos quais destacamos os relacionados ao transplante hepático pediátrico..$^{3-4,6}$

No entanto, diferente dos avanços tecnológicos, estudos ressaltam que pouco se tem estudado sobre o impacto emocional da experiência do transplante sobre a criança e sua família. ${ }^{7-10} \mathrm{~A}$ família, diante da experiência do transplante, necessita alterar todo o seu funcionamento para atender às suas demandas e, sendo a doença um evento familiar, partimos da premissa que esta deve ser incorporada ao cuidado dos profissionais.

Para tanto, é importante que os profissionais estejam equipados e preparados para desempenhar uma prática baseada em evidências. Assim sendo, perguntamos: o que tem sido pesquisado e publicado sobre as famílias de crianças transplantadas, na última década? Quais os resultados encontrados e quais novos caminhos de pesquisa poderiam ser abordados de forma a colaborar com essa área de conhecimento? 
Mediante tais indagações, realizamos o presente estudo, com o objetivo de explorar o conhecimento disponível na literatura de Ciências da Saúde da última década sobre famílias de crianças submetidas a transplante de órgãos, bem como identificar lacunas nessa área de conhecimento, sugerindo caminhos para pesquisas futuras.

\section{MÉTODOS}

Trata-se de um estudo exploratório de revisão da literatura, cujas etapas operacionais basearam-se no "Curso de Pesquisa Bibliográfica: Medline, Lilacs, Portal de Revistas Científicas CAPES", promovido pela Escola Paulista de Medicina da Universidade Federal de São Paulo. A estratégia metodológica apoiou-se no estudo de revisão sistemática. ${ }^{13}$

A questão básica que norteou a investigação é: o que tem sido estudado e pesquisado a respeito da família da criança transplantada?

Utilizando-se as bases de dados LILACS (Literatura LatinoAmericana e do Caribe em Ciências da Saúde), MEDLINE (Medical Literature Analysis and Retrieval System - online) e PUBMED fizemos uma busca por estudos relacionados ao tema de estudo publicados no período entre 1996-2006, utilizando-se as palavras chave: child (criança), transplantation (transplante) e family (família).

Cabe ressaltar que a palavra nursing (enfermagem) foi excluída da busca, pois não apareceram resultados associados, indicando-nos de antemão uma necessidade emergente de estudos de Enfermagem sobre o tema. Procedemos então à revisão sistemática, sem especificação de uma área nas Ciências da Saúde.

Ao todo, 52 referências foram encontradas, sendo desconsideradas aquelas cujos títulos ou resumos permitiram antecipadamente concluir que não se relacionavam especificamente com a questão deste estudo. Passou-se à busca da publicação completa das remanescentes em bibliotecas especializadas, tendo sido encontrados onze estudos completos. Destes, dois tratavam de famílias de adultos transplantados e foram desconsiderados, totalizando nove estudos para análise.

Os trabalhos foram classificados quanto ao tipo de estudo, ${ }^{14}$ ano e local de realização, objetivos, resultados e principais conclusões.

\section{RESULTADOS}

Os resultados encontrados indicam que a maioria $(60 \%)$ dos estudos tem sido publicada a partir dos últimos cinco anos (Gráfico 1), é de abordagem quantitativa (Gráfico 2) e é originária da Europa ou da América do Norte (Tabela 1).

Gráfico 1: Ano de Publicação

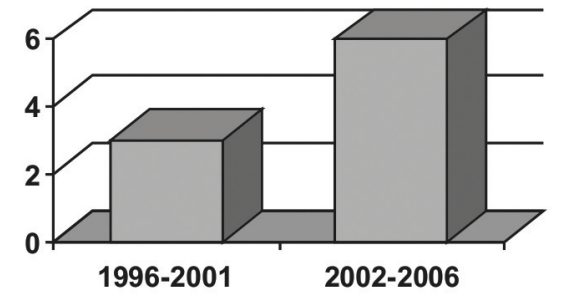

Gráfico 2: Abordagens de Pesquisa

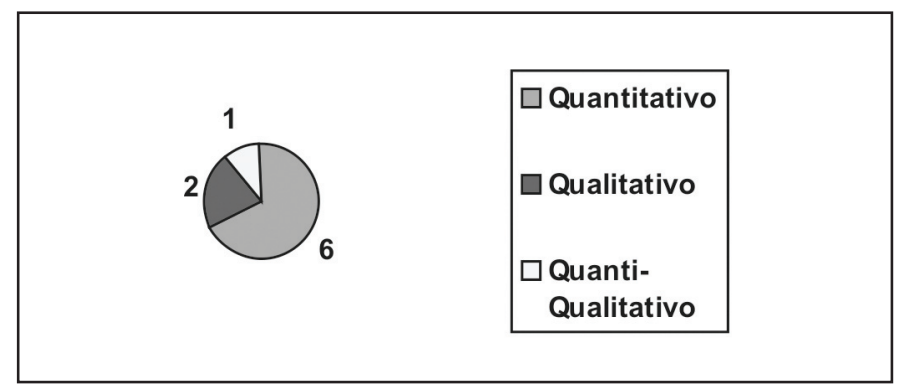

Tabela 1. País de Origem

\begin{tabular}{cc}
\hline País de origem & Número de trabalhos encontrados \\
\hline Estados Unidos & 4 \\
França & 1 \\
Inglaterra & 2 \\
Itália & 1 \\
Suécia & 1 \\
\hline
\end{tabular}

Em relação aos objetivos, resultados e conclusões, a análise dos textos permitiu separá-los em quatro temas distintos: Funcionamento e Adaptação Psicossocial, Experiência familiar, Qualidade de vida e Indicadores de prognóstico. Os temas e textos são apresentados a seguir.

\section{Funcionamento e Adaptação Psicossocial}

Três dos textos selecionados estão voltados à exploração da percepção do funcionamento psicossocial de crianças submetidas a transplante, bem como o funcionamento psicossocial dos pais. ${ }^{15-17}$ Os resultados mostram que os pais geralmente fazem uma avaliação mais negativa em relação à criança do que ela própria, o que indica, por parte deles, uma visão hipervigilante em relação à criança transplantada, ${ }^{15}$ indicando também, alterações negativas nos relacionamentos conjugais e familiares, bem como um elevado índice de depressão e estresse pós-traumático dos pais, relacionados ao distanciamento de relações sociais e sentimentos de desesperança e incerteza quanto ao futuro. ${ }^{15-16}$

Estes estudos trazem evidências de que a forma como o transplante é percebido pela família influencia na forma como ela lida com a experiência, e que a adaptação da criança ao transplante é estreitamente relacionada ao funcionamento familiar; disfunções familiares aumentam as chances de problemas psicológicos na adaptação da criança no pós-transplante. ${ }^{16-17}$

Tais trabalhos reforçam que compreender o transplante pediátrico como um evento da família e analisar suas implicações na dinâmica familiar é um caminho de pesquisa que ainda precisa ser explorado, para que os profissionais de saúde consigam propor intervenções preventivas para famílias que estão atravessando a experiência. Destacam ainda que a gravidade clínica do paciente é menos importante do que o funcionamento familiar para 
determinar a adaptação pós-transplante, sugerindo mais estudos que enfoquem o cotidiano da família nas diferentes fases da experiência do transplante. ${ }^{15-17}$

Outro estudo selecionado buscou investigar as marcas psicológicas presentes na criança que passou pela experiência de possuir uma doença hepática crônica na primeira infância e depois foi submetida a transplante hepático. ${ }^{18}$

Os resultados indicam a experiência do transplante sempre muito presente na vida da criança, que mesmo em sessões de desenho livre, traz à tona a experiência, representando mesas cirúrgicas, aparelhos, instrumentos, ou duas crianças lado a lado - uma saudável e outra bem doente. Os maiores medos dessas crianças estão relacionados à morte $(61 \%$ dos pacientes e $11 \%$ dos controles) e a serem abandonadas pelos pais (50\% dos pacientes e $27,7 \%$ dos controles). Mais da metade dos pais afirmou que o transplante e a doença hepática trouxeram impacto negativo para o desenvolvimento intelectual e emocional da criança.

Além disso, um ponto neste estudo merece especial atenção: os pais relatam que falar sobre o transplante com a criança é muito difícil para eles. Dentre os principais motivos para isso estão: a preocupação com a resposta emocional da criança, o fato de que os pais consideram o transplante um assunto constrangedor e delicado de ser abordado e o evento da morte do doador como sendo algo muito difícil de explicar para a criança. Diante disso, o estudo mostra também que quase $90 \%$ dos pais não conseguem nem imaginar as fantasias que seus filhos têm sobre o transplante, visto que esse assunto não é discutido em família. Os dados sugerem que quando não há comunicação sobre o transplante da criança entre os familiares, a criança pode passar pela experiência de forma traumática, como se houvesse um "vazio" em sua mente, e infere que o tema pode estar sendo excluído das conversas da família por razões culturais e psicológicas, que merecem maiores estudos.

\section{Experiência familiar}

Três estudos focam os pais e demais membros da família durante a experiência do transplante, buscando compreender a experiência de espera pelo transplante da criança, bem como suas alterações na dinâmica familiar durante essa experiência ${ }^{18-19}$ e posterior convívio com a criança transplantada. ${ }^{20}$

Durante a experiência de aguardar o transplante, dados quantitativos indicam que as mães apresentam maior grau de ansiedade, disfunção social, sintomas somáticos e depressão do que os pais. ${ }^{20}$ Os dados qualitativos indicam que as categorias representativas do período de espera pelo transplante são: colocando a vida em espera, experimentando escassez de suporte emocional, estabelecendo uma nova rede de suporte, sofrendo mudanças de papéis, preocupando-se com a situação financeira e procurando tirar o melhor da situação. ${ }^{19}$

Em relação aos sentimentos dos pais durante a experiência do transplante, a principal categoria apresentada no estudo foi a de incerteza quanto ao futuro. Com a experiência do transplante, os pais referem que a vida deles mudou completamente, principalmente no sentido de estabelecer novas prioridades e valores na vida; percebem a medicação como sendo a salvação da criança e sentem muito medo de esquecer os horários corretos da administração desta; sentem que perderam muitos amigos devido às limitações de convívio social que agora vivenciam e sentem-se incompreendidos por eles. Em relação à comunicação com a criança, muitos pais relataram sentir medo de abordar assuntos relativos ao transplante com ela, evitando falar sobre o assunto. ${ }^{20}$
Tais estudos reforçam a importância da avaliação familiar pela equipe de saúde, de forma a oferecer novos recursos para que a família passe pela experiência ampliando sua rede de suporte social, e sugerem outros estudos que envolvam a avaliação da comunicação da família com a criança sobre o transplante.19,20 Ressaltam a importância de integrar o cuidado da família ao cuidado da criança na experiência de transplante e sugerem estudos que acessem sistematicamente a adaptação da criança/família para que se possa prover suporte e aconselhamento adequados durante todas as etapas do processo. ${ }^{20}$

\section{Qualidade de vida}

Um estudo selecionado está voltado à qualidade de vida de crianças submetidas a transplante hepático ou renal sob a perspectiva delas, de seus pais e dos profissionais de saúde. ${ }^{21}$

Os resultados são preocupantes. As crianças transplantadas indicaram menor satisfação em relação à autopercepção, atividades escolares e saúde. As mães das crianças transplantadas demonstram necessidade intensa de apoio emocional para si próprias e sentem falta desse suporte também para seus filhos. A qualidade de vida das mães é alterada consideravelmente, principalmente porque elas deixam de trabalhar e alteram suas relações sociais por ficarem o tempo todo com o filho transplantado.

$\mathrm{O}$ estudo reforça a necessidade de se pensar em alternativas para que a criança atravesse o período pós-transplante com as menores alterações possíveis na sua rotina, adotando um novo estilo de vida que possa ser compatível com a nova realidade. Para tal, é imperativo que criança e família entrem em contato com novos recursos e formas de manejo e adaptação.

\section{Indicadores de prognóstico}

Um trabalho destina-se a examinar diferenças físicas, demográficas e psicológicas entre dois grupos de famílias de crianças com fibrose cística: aquelas que sobreviveram ao período de espera pelo transplante e as que não sobreviveram, verificando se existe relação entre os fatores anteriormente mencionados e o tempo de sobrevivência pós-transplante. ${ }^{22}$

Os maiores índices de estresse foram encontrados em famílias de crianças que não sobreviveram ao transplante. Avaliou-se que um total de $20 \%$ das famílias participantes tinham um funcionamento caótico e tal fato está relacionado a um período mais curto de sobrevivência pós-transplante. Houve relação estatisticamente significante entre o maior tempo de sobrevida pós-transplante e a idade (quanto mais jovem, maior a sobrevida) e a gravidade clínica (quanto mais grave no pré-transplante, maior a sobrevida no pós-transplante).

Não há evidência sugestiva neste estudo de que fatores psicossociais devam de fato ser usados como contra-indicação ao transplante. $\mathrm{O}$ estudo sugere que novos estudos sejam feitos, com um olhar voltado à identificação de fatores que possam ser associados à resiliência familiar de forma a prolongar a sobrevida após o transplante.

\section{DISCUSSÃO}

Diante dos resultados apresentados, percebemos que independentemente dos temas abordados, as pesquisas têm focado de maneira geral as diferentes etapas do processo que envolve o transplante de órgãos, 
seja na fase da espera pelo transplante no momento da cirurgia e no pós-operatório imediato, bem como a convivência social da criança transplantada, principalmente com os pais.

No entanto, podemos verificar que apenas duas pesquisas enfocam a família como um sistema. Destas, uma objetivou a verificação de uma possível associação entre o funcionamento familiar e o prognóstico da criança após o transplante 17 e outra se destinou à investigação sobre o que acontece com outros membros da família enquanto aguardam o transplante da criança. ${ }^{20}$

As demais pesquisas estão relacionadas às percepções isoladas da situação de transplante seja da criança, de um dos pais, do subsistema conjugal, ou ainda dos profissionais de saúde.

Quando retomamos a perspectiva de que a doença é um evento da família, ${ }^{12}$ pensamos nesta como sendo um grupo de indivíduos em intensa e constante interação, e, portanto, a doença no contexto familiar não pode ser considerada sob o ponto de vista de apenas um dos membros do sistema, mas sim sob a perspectiva do sistema como um todo, que é mais do que a soma dos seus membros. ${ }^{23}$

Não foram encontrados nos procedimentos de revisão adotados estudos que tivessem essa abordagem no cuidado com a família, considerando aspectos inerentes à dinâmica familiar e às diferentes formas de manejo e adaptação que o sistema familiar desenvolve na experiência do transplante pediátrico. Tal direcionamento de pesquisa se faz necessário para que seja compreendido o funcionamento familiar de uma criança que é submetida a um transplante de órgãos e para que se possa avaliar e propor intervenções de Enfermagem a essas famílias.

Atualmente, em diversas áreas dentro da Enfermagem tem crescido o número de trabalhos qualitativos feitos com o intuito de desenvolver e disseminar o conhecimento de fenômenos não mensuráveis por meio de análises estatísticas, primariamente os complexos fenômenos das interações humanas. Uma das áreas que merece destaque é a Enfermagem da Família, que tem como objetivo compreender as demandas da família nos diferentes contextos do processo saúde-doença e, com isso, criar estratégias para o cuidado desta. Diversos estudos já têm sido realizados nesse sentido em diferentes momentos do processo saúde-doença ${ }^{24-26} \mathrm{e}$ dentro do contexto de doação de órgãos. ${ }^{27-28} \mathrm{~A}$ imersão nessa área de conhecimento, associada à ausência de estudos qualitativos na área de transplante, sugere novos caminhos e abordagens de pesquisa na Enfermagem em âmbito nacional.
Os resultados apresentados em todos os estudos destacam a importância do apoio às famílias de crianças transplantadas em todas as etapas da experiência de transplante. Independentemente do momento em que a família se encontra vivendo a experiência, estudos sugerem que a necessidade de apoio e de informações quanto ao estado de saúde da criança é de fundamental importância, e que o fato de a criança estar em boas condições clínicas não significa que os pais não tenham mais preocupações em relação ao seu estado de saúde, já que a experiência do transplante foi definida como uma experiência de intensa e constante incerteza. ${ }^{20}$

Tais dados convergem com um estudo que objetivou compreender a percepção dos pais das crianças que passam pela experiência do transplante de pulmão. $O$ estudo não foi encontrado na busca sistemática, mas é apontado como uma das principais obras que buscou compreender a percepção do subsistema conjugal diante da experiência. ${ }^{29}$ Apesar de não fornecer dados sobre os demais membros da família, os autores trazem ricas contribuições no que diz respeito às demandas dos pais em meio à experiência, e não poderia deixar de ser mencionado como um referencial para aprofundamento das questões levantadas nos estudos analisados neste trabalho.

Não foram encontrados estudos que abordassem caminhos para intervenções de Enfermagem com essas famílias, embora já haja reconhecimento da necessidade de apoio em todas as etapas da experiência de transplante. Estudos que desenvolvam e validem intervenções junto a essas famílias podem trazer um avanço importante ao cuidado em meio à experiência de transplante.

\section{RECOMENDAÇÕES}

A literatura analisada apresenta dados consistentes acerca do grande impacto que a experiência do transplante da criança exerce sobre todos os membros da família. Considerando a família como um sistema que envolve, além da soma de seus membros, a complexidade de seu funcionamento e as interações que se processam a todo o momento em seu cotidiano, acreditamos ser de suma importância o desenvolvimento de estudos qualitativos que enfoquem a natureza e a dinâmica dessas interações no contexto de uma família que passa pela difícil experiência de ter uma criança submetida a um transplante de órgãos, para que possamos chegar a um entendimento mais completo sobre as diferentes interfaces da experiência do transplante pediátrico, e possamos, como enfermeiros e demais profissionais de saúde, atender às demandas da família.

\section{ABSTRACT}

This study aimed to explore the available knowledge in the scientific literature regarding the experience of families of children who are transplant recipients, and to identify gaps in that area of knowledge. A systematic review of the literature was performed by searching in the Medline, Pubmed and Lilacs database using the keywords child, transplantation and family. Nine texts were selected to the analysis, which were organized in terms of type of study, year and country of origin, purposes, methodological steps, and results. Data analysis allowed the identification of four main categories that have been discussed in the literature: Functioning and Psychosocial adaptation, family experience, quality of life, prognostic indicators; every selected article had been published in foreign countries. In terms of conclusion, it was established that the literature researched already shows solid data proving the great impact that the transplant experience has on the child and the family. However, future research focusing the experience of the family in terms of its functioning within the transplant experience and the Nursing intervention approach focused in these families, is imperative.

Keywords: Review Literature as Topic; Transplants; Family; Family Relationship. 


\section{REFERÊNCIAS BIBLIOGRÁFICAS}

1. Pereira WA. Manual de Transplantes de Órgãos e Tecidos. 2 ed. Rio de Janeiro: Medsi, 2000.

2. Kurz JMcC. Vulnerability of well spouses involved in lung transplantation. J Fam Nurs. 2002;8(4):353-70

3. Otte JB. History of pediatric liver transplantation. Where are we coming from? Where do we stand? Pediatr Transplantation. 2002;6:378-87.

4. Mir S, Erdogan H, Serdaroglu E, Kabasakal C, Hoscoskun C. Pediatric renal transplantation: single center experience. Pediatr Transplantation. 2005;9:56-61.

5. Hsu T. Biological and psychological differences in the child and adolescent transplant recipient. Pediatr Transplantation. 2005;9:416-21.

6. Tan N, Dhawan A. Pediatric Liver Transplantation: Review of literature 2003-2005 Pediatr Transplantation. 2006;10:549-57.

7. Wise BV. In their own words: the lived experience of pediatric liver transplantation. Qual Health Res. 2002;12(1):74-90.

8. Moore KA. Liver Transplantation: what do we really know about the long-term impact? Liver Transpl. 2003;9(11):1149-51.

9. Brosig CL. Psychological functioning of pediatric lung transplant candidates/ recipients: a review of the literature. Pediatr Transplantation. 2003;7:390-94.

10. Falkenstein K. Proactive psychosocial management of children and their families with chronic liver disease awaiting transplant. Pediatr Transplantation. 2004;8:205-7.

11. Mendes AMC, Bousso RS. Compreendendo a dinâmica familiar da criança submetida a transplante de órgãos. Rev. Soc Bras Enferm Ped. 2006;6(1):5-8.

12. Wright LM; Leahey M. Enfermeiras e Famílias: um guia para avaliação e intervenção na família. 3 ed. São Paulo: Roca, 2002.

13. Galvão CM, Sawada NO, Trevizan MA. Revisão sistemática: recurso que proporciona a incorporação das evidências na prática de enfermagem. Rev Latino-Am. Enfermagem. 2004;12(3):549-56.

14. Polit DF, Beck CT, Hungler BP. Fundamentos de Pesquisa em Enfermagem: métodos, utilização e avaliação. 5 ed. São Paulo: Artmed, 2004.

15. Sudan D, Horslen S, Botha J, Grant W, Torres C; Shaw Jr B et al. Quality of life after Pediatric Intestinal Transplantation: the perception of pediatric recipients and their parents. Am J of Transplant. 2004;4:407-13.

16. Young GS, Mintzer LL, Seacord D, Castañeda M, Mesrkhani V, Stuber ML. Symptoms of posttraumatic stress disorder in parents of transplant recipients: incidence, severity, and related factors. Pediatrics. 2003;111(6):725-31.

17. DeMaso DR, Kelley SD, Bastardi H, O'Brien P, Blume ED. The longitudinal impact of psychological functioning, medical severity, and family functioning in pediatric heart transplantation. J Heart Lung Transplant. 2004;23:473-80.

18. Gritti A, Di Sarno AM, Comito M, De Vincenzo A, De Paola P, Vajro P. Psychological impact of liver transplantation on children's inner worlds. Pediatr Transplantation 2001;5:37-43.

19. Stubblefield C, Murray RL. Waiting for lung transplantation: family experiences of relocation. Pediatr Nurs. 2002;28(5):501-4.

20. Serrano-Ikos E, Lask B. Psychosocial morbidity in children, and their families, awaiting heart or heart-lung transplantation. J Psychosom Res. 1997;42(7):253-60.

21. Manificat S, Dazord A, Cochat P, Morin D, Plainguet F, Debray D. Quality of life of children and adolescents after kidney or liver transplantation: child, parents and caregiver's point of view. Pediatr Transplantation. 2003;7:228-35.

22. Serrano-Ikos E, Lask B. The psychosocial correlates of transplant survival. J Cyst Fibros 2003;2:49-54.

23. Angelo M. Com a família em tempos difíceis: uma perspectiva de Enfermagem. (tese). São Paulo: Escola de Enfermagem da USP; 1997.

24. Bousso RS. Buscando preservar a integridade da unidade familiar: a família vivendo a experiência de ter um filho na UTI Pediátrica. (tese). São Paulo: Escola de Enfermagem da USP; 1999.

25. Pettengill MAM. Vulnerabilidade da família: desenvolvimento do conceito. (tese). São Paulo: Escola de Enfermagem da USP; 2003.

26. Rossato LM. Cuidando para a criança crescer apesar da dor: a cotidiano da família da criança com artrite reumatóide juvenil.(tese). São Paulo: Escola de Enfermagem da USP; 2003.

27. Sadala MLA. Doação de órgãos: a experiência de enfermeiras, médicos e familiares de doadores. São Paulo: Editora Unesp, 2004.

28. Bousso RS. Um tempo para chorar: a família dando sentido à morte prematura do filho(tese). São Paulo: Escola de Enfermagem da USP; 2006.

29. Stubblefield C, Murray RL. Parent's perceptions of their children's lung transplant experience. J Fam Nurs. 1998;4:367-86. 